\title{
Embedded System based Voting Machine System using Wireless Technology
}

\author{
Dhinesh Kumar.M ${ }^{1}$, Santhosh.A ${ }^{2}$, Aranganadhan.N.S ${ }^{3}$, Praveenkumar. ${ }^{4}$ \\ Final year, B.E (Electrical and Electronics Engineering),CSI College of Engineering, The Nilgiris,TamilNadu ${ }^{1,2,3,4}$
}

\begin{abstract}
The objective of voting is to allow voters to exercise their right to express their choices regarding specific issues, pieces of legislation, citizen initiatives, constitutional amendments, recalls and/or to choose their government and political representatives. But, now- a -days it has become very usual for some forces to indulge in rigging which may eventually lead to a result contrary to the actual verdict given by the people. In order to provide inexpensive solutions to the above, this project will be implemented with biometric system i.e. finger print scanning. This is used to ensure the security to avoid fake, repeated voting etc. It also enhances the accuracy and speed of the process. The system uses thumb impression for voter identification as we know that the thumb impression of every human being has a unique pattern. Thus it would have an edge over the present day voting systems. The purpose of such system is to ensure that the voting rights are accessed only by a legitimate user and no one else. In this, creation of a database consisting of the thumb impressions of all the eligible voters in a constituency is done as a pre-poll procedure. During elections, the thumb impression of a voter is entered as input to the system. This is then compared with the available records in the database. If the particular pattern matches with anyone in the available record, access to cast a vote is granted. But in case the pattern doesn't match with the records of the database or in case of repetition, access to cast a vote is denied or the vote gets rejected. The result is instantaneous and counting is done. The overall cost for conducting elections gets reduced and so does the maintenance cost of the systems. The postal type of voting is not convenient for everyone, Hence a new option like vote from any places in Tamil Nadu by using the pre saved details of AADHAR card and by choosing the specific area for voting it comes in handy so that it becomes easy for the non home persons to vote from any place inside Tamil Nadu. The age is verified before voting and if the voter does not vote for more than three elections the citizenship will be canceled. The disabled persons will have a swipe machine where they will use their AADHAR card to vote. These simulations results are verified with the help of Keil vision.
\end{abstract}

Index Terms: Finger print scanning, Database, Biometric system, Microcontroller.

\section{I.INTRODUCTION}

After getting freedom from British government, Indian Government provide a right to Indian people to elect their interested leader. For conducting and controlling voting in India, a separate commission was introduced. Which was named as Election Commission of India(ECI). This commission is not favorable or support to any political party. As per rules which have been in law, this commission works. For the persons, who have age of 18 and above are eligible to enroll their vote. To cast their vote, Election commission provides voter id cards. From last few decades paper voting procedure is going on. After polling votes in polling booths, Those paper format votes have been placed in ballot boxes. That entire box placed in the rooms of schools or colleges. If those rooms are perfect with interior walls, then there is no problem. If those inner walls are older one, then ants will damage that valuable ballot box. At this instant, Election commissions need to conduct elections at that particular place once again. To avoid this problem, Author going to use Evoting system.

\section{II.LITERATURE SURVEY}

Umang Shah, Trupt Sha et al these papers attempt to solve this problem. Today we all have AADHAR CARD so the Government has all the data base of us including finger print and retina. As we all know that general election happened last year but no one really talks about the use of
AADHAR CARD. So if we used that database effectively with the help of micro controller then we can completely overcome the problem of bogus voting. This paper shows how this problem can be solved with the help of combination of biometric finger print scanner and micro controller [1].

Pooja Chugh et al this paper tells about Voting is a decision making mechanism in a consensus-based society and security is most essential part of voting. The critical role in determining the outcome of an election, Electronic Voting Systems must be designed and developed with the greatest care. It is claimed by the Indian Election Authorities that paperless Electronic Voting Systems suffer from significant vulnerabilities. Even brief access to the machines could allow dishonest election insiders or other frauds to alter election results. In this paper, an idea is proposed to upgrade the existing voting system that is based on complete biometric traits of voter which are saved in a government database as Aadhar (U-id) number database. These biometrics traits provide secure and feasible authentication to the voters, thus preventing the fraud and illegal voting [2].

Shafi'í Muhammad Abdulhamid et al this paper, we developed an electronic voting system, which will eliminate rigging and manipulation of results to its barest minimum, this problem is mostly associated with the manual system of voting. The implementation of 
electronic voting system in Nigeria will boost the integrity of INEC and the result they produce. The programs used to develop this system are PHP, MySQL, Java Query, CSS and HTML. These packages make the Graphic Interface User friendly enough for even those with little or no computer knowledge [3].

Sanjay Kumar et al Fingerprint biometric is the most widely deployed publicized biometrics for identification. This is largely due to its easy and cost effective integration in existing and upcoming technologies. The integration of biometric with electronic voting machine undoubtedly requires less manpower, save much time of voters and personnel eliminate rigging, ensure accuracy, transparency and fast results in election. In this paper, a framework for electronic voting machine based on biometric verification is proposed and implemented. The proposed framework ensures secured identification and authentication processes for the voters and candidates through the use of fingerprint biometrics [4].

B. Madan Mohan Reddy et al this paper proposes a method for safe and secure aadhaar based biometric voting system to avoid misconceptions which are going to takes place in elections. From last few years in India, aadhaar enrollment process is going on in Andhra Pradesh. At that time, Indian government collects details of finger prints and IRIS of every person. This entire data of each and every person persists in the database of Indian government.

If Indian Government link this database to authors proposed method, Indian Government no need to collect details of finger print of every person. So, if Indian Government adopt biometric voting system for voting purpose, we can easily avoid rigging in elections. One more advantage of this project is, if an alcoholic person enters into polling booth, buzzer will alert authorized persons or constables who are in election duty. Because of Alcoholic sensor, we can provide peaceful environment at polling booth. If an unauthorized person enters into polling booth to cast his vote, buzzer will alert booth level officer. If already vote casted person enters into booth with his RFID tag for 2nd time voting, then also buzzer will alert booth level officer [5].

M.Sudhakar et al this paper focuses on simple, low cost fingerprint based electronic voting machine using ARM9 microcontroller. An electronic voting system is a voting system in which the voters' and voting data is recorded, stored and processed digitally. The proposed system consists of controller hardware and software. The hardware is implemented with ARM9 microcontroller along with KY-M6 finger-print module. The software code is developed in WINCE6 development environment for interfacing the ARM processor with finger-print module. The proposed system gives the best solution for minimizing the time taken for identifying the voter. The design implemented in the FP-EVM is portable, flexible and with minimum power consumption. The designed system is user-friendly, easily adaptable and costeffective. Further, the designed system has simple architecture, fast response time and scope for further expansion [6].
Rohan Patel et al this project proposes a secure online evoting system that uses UIDAI or aadhar database as its backend. The system ensures authentication of an individual by matching fingerprints and eligibility is checked by calculating the age of the voter thus making the existing voting cards redundant. The proposed system can handle voting at different levels such as Parliamentary, Municipality, State legislative assembly, etc simultaneously. The project will bring transparency in the voting process by assuring the voters that their votes will be in favor of the candidates of their choice. Besides electronic recording and counting of votes will be faster, more accurate and less labor intensive. The design of this system will make voting process more convenient and may therefore lead to improve the turnout [7].

\section{III.EXISTING SYSTEM}

There are two types of problems with EVM which is currently in use:

1. Security Problems - One can change the program installed in the EVM and tamper the results after the polling. By replacing a small part of the machine with a look-alike component that can be silently instructed to steal a percentage of the votes in favor of a chosen candidate. These instructions can be sent wirelessly from a mobile phone.

2. Illegal Voting (Rigging) - The very commonly known problem, Rigging which is faced in every electoral procedure. One candidate casts the votes of all the members or few amount of members in the electoral list illegally. This results in the loss of votes for the other candidates participating and also increases the number votes to the candidate who performs this action. This can be done externally at the time of voting.

\section{IV.PROPOSED SYSTEM BLOCK DIAGRAM}

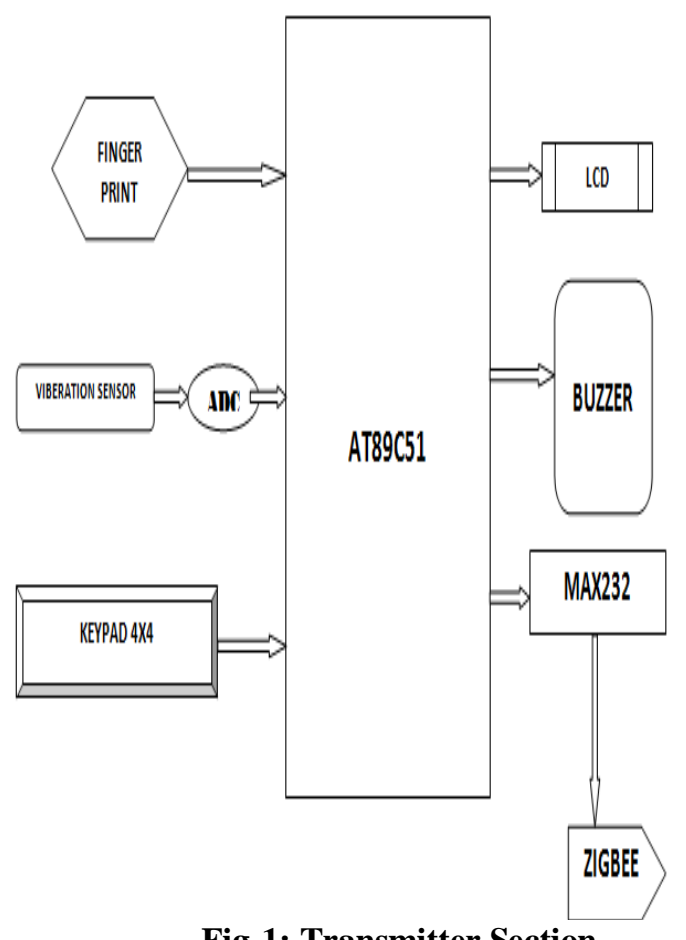

Fig-1: Transmitter Section 


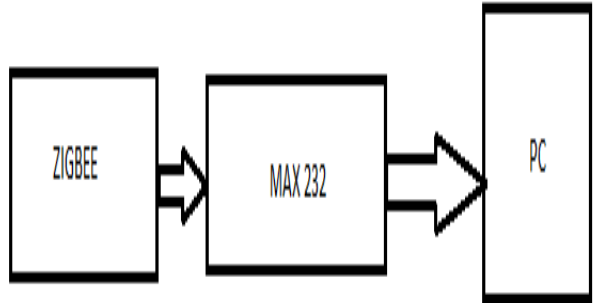

Fig-2: Receiver Section

\section{ADVANTAGES}

1. The system is highly reliable, tamper-proof and secure.

2. In the long run the maintenance cost is very less when compared to the present systems.

3.Illegal practices like rigging in elections can be checked for.

4. It is possible to get instantaneous results and with high accuracy.

\section{V.HARDWARE DESCRIPTION}

\section{A.Zigbee}

ZigBee is a mesh network specification for low-power wireless local area networks (WLANs) that cover a large area. ZigBee was designed to provide high data throughput in applications where the duty cycle is low and low power consumption is an important consideration. (Many devices that use ZigBee are powered by battery.) Because ZigBee is often used in industrial automation and physical plant operation, it is often associated with machine-to-machine (M2M) communication and the Internet of Things (IoT)

\section{B. Vibration Sensor}

A transducer, such as that incorporating a laser or a piezoelectric crystal, which converts vibrations into an electrical equivalent such as a voltage. However subtle and unnoticed by human senses and it is a telltale sign of machine condition. Abnormal vibration indicative of problems with an industrial machine can be detected early and repaired before the event of machine failure; because such a failure is potentially costly in terms of time, cost, and productivity, vibration measurement allows industrial plants to increase efficiency and save money. Therefore, vibration analysis is used as a tool to determine equipment condition as well as the specific location and type of problems.. Vibration sensors are simply packaged as a raw sensor. Vibration transducers are slightly more complex sensors which output a voltage or current signal.

\section{C.Microcontroller}

A microcontroller is a small computer ( $\mathrm{SoC})$ on a single integrated circuit containing a processor core, memory, and programmable input/output peripherals. Program memory in the form of Ferroelectric RAM, NOR flash or OTP ROM is also often included on chip, as well as a typically small amount of RAM. Microcontrollers are designed for embedded applications, in contrast to the microprocessors used in personal computers or other general purpose applications consisting of various discrete chips.

\section{D.Buzzer (Horn)}

A buzzer or beeper is an audio signaling device, which may be mechanical, electromechanical, or piezoelectric. Typical uses of buzzers and beepers include alarm devices, timers and confirmation of user input such as a mouse click or keystroke. While technological advancements have caused buzzers to be impractical and undesirable, there are still instances in which buzzers and similar circuits may be used.

\section{E. $L C D$}

A liquid-crystal display (LCD) is a flat-panel display or other electronic visual display that uses the lightmodulating properties of liquid crystals. Liquid crystals do not emit light directly.LCDs are used in a wide range of applications including computer monitors, televisions, instrument panels, aircraft cockpit displays, and signage. They are common in consumer devices such as DVD players, gaming devices, clocks, watches, calculators, and telephones, and have replaced cathode ray tube (CRT) displays in nearly all applications. They are available in a wider range of screen sizes than CRT and plasma displays, and since they do not use phosphors, they do not suffer image burn-in. LCDs are, however, susceptible to image persistence. The LCD screen is more energy-efficient and can be disposed of more safely than a CRT. Its low electrical power consumption enables it to be used in battery-powered electronic equipment more efficiently than CRTs. It is an electronically modulated optical device made up of any number of segments controlling a layer of liquid crystals and arrayed in front of a light source (backlight) or reflector to produce images in color or monochrome.

\section{F.Matrix keypad}

Most electronic keyboards used in synthesizers, electronic organs, and digital pianos use a keyboard matrix circuit in which eight rows and eight columns of wires cross. In this way, 16 wires can provide (8x8) 64 crossings - sufficient for a full five octaves of range (61 notes). By scanning these crossings, a keyboard controller can determine which key was pressed. Monophonic instruments and most low-cost computer keyboards reduce costs by leaving out most or all of those diodes. To avoid "phantom keys", the keyboard controller in modern low-cost computer keyboards will ignore further key presses once two keys (other than modifier keys) have been pressed, which is known as jamming.

\section{G.Fingerprint Identification Module}

Fingerprint processing includes two parts: fingerprint enrollment and fingerprint matching (the matching can be 1:1 or 1:N). When enrolling, user needs to enter the finger two times. The system will process the two time finger images, generate a template of the finger based on processing results and store the template. When matching, user enters the finger through optical sensor and system will generate a template of the finger and compare it with templates of the finger library. For 1:1 matching, system will compare the live finger with specific template designated in the Module; for 1:N matching, or searching, system will search the whole finger library for the matching finger. In both circumstances, system will return the matching result, success or failure. 
INTERNATIONAL JOURNAL OF INNOVATIVE RESEARCH IN ELECTRICAL, ELECTRONICS, INSTRUMENTATION AND CONTROL ENGINEERING Vol. 4, Issue 2, February 2016

\section{DESCRIPTION}

- $\quad$ Power DC 4.5V-6.0V

- Interface UART(TTL logical level)/ USB 1.1

- Working current Typical: 100mA Peak:150mA

- Matching Mode 1:1 and 1:N

- Image acquiring time< $0.8 \mathrm{~s}(1: 880)$

- Window dimension $18 \mathrm{~mm} * 22 \mathrm{~mm}$

\section{VI.SOFTWARE DESCRIPTION}

The proposed system has implemented Keil C development tools for the ARM family to support every level of developer from the Professional applications engineer to the student just learning about embedded software development. The industry standard Keil C compilers, Macro assemblers, Debuggers, Real time kernels, and single board computers support all compatible derivatives and help you to get your projects completed on schedule. With the Keil tools, we can generate embedded applications for virtually every ARM process applications. Keil $\mathrm{C}$ vision 4 help provide the variation simulation output.

\section{A.PROGRAM:}

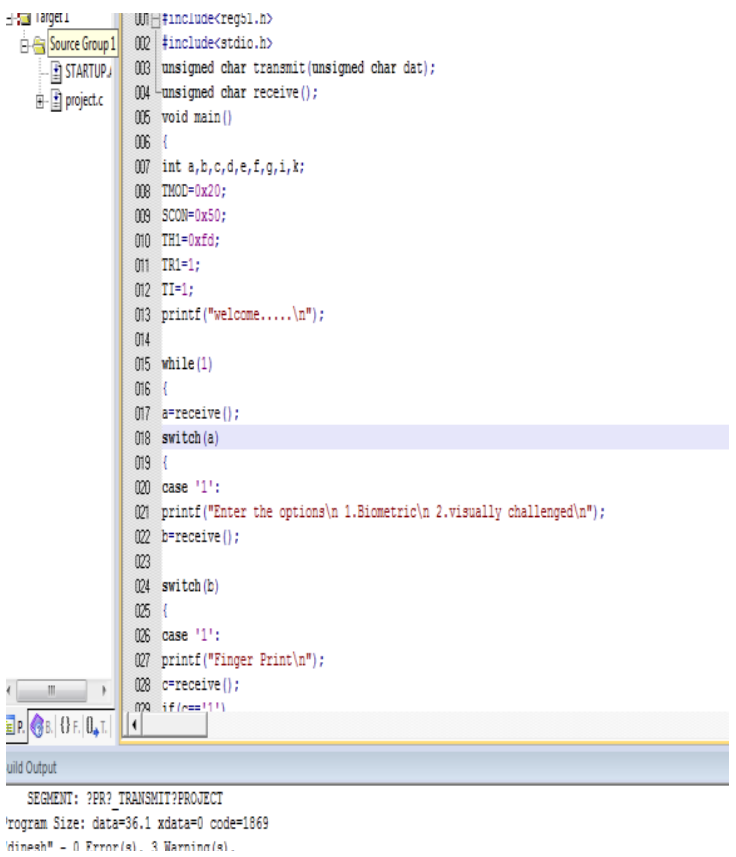

Fig-3: Program for Proposed System

\section{B.SIMULATION RESULTS:}

\section{UART \#1}

welcome.....

inter the option

1.Biometric

2.visually challenged

Finger Print

Finger print Matched

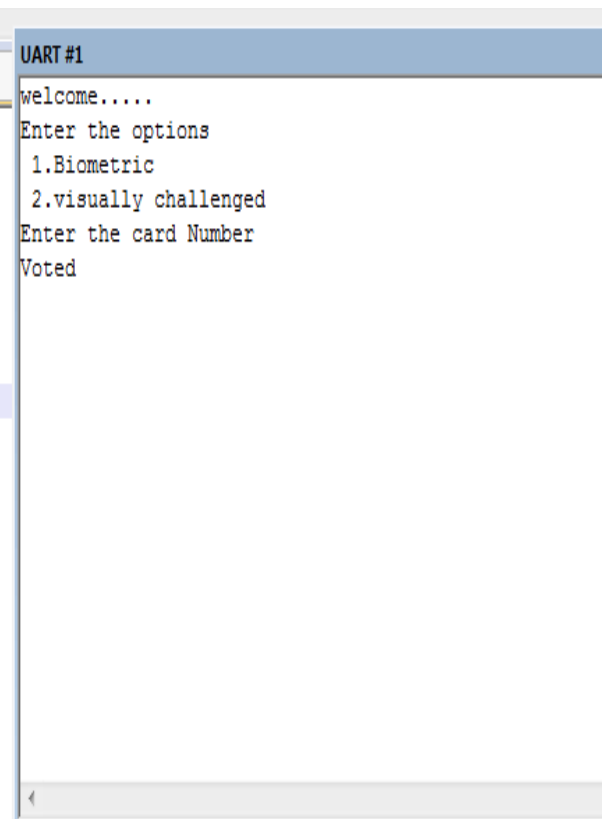

Fig-4: Simulation Results

\section{VII.CONCLUSION}

This project can be used for voting since it overcome all the draw backs of ordinary voting machine also provide additional security. Its main advantage is that since fingerprints of every person is unique and hence this system completely reduces the chance of invalid votes. The system can be manufactured simply as well as cheap and casting vote becomes easier by the process of voting from any place inside Tamilnadu.

\section{REFERENCES}

1. Umang Shah, Trupt Shah, Marteen Kansagara, Saagar Daxini,(March 2015),Biometric Secured Voting Machine to Avoid Bogus Voting Based on AADHAR CARD.

2. Patil Rahul H, Tarte Babita B, Wadekar Sapana S, Zurunge Bhakti S,Prof. Phursule R, (September 2015), A Secure E-Voting System Using Face Recognition and Dactylogram.

3. Pradeep Dimri, Pooja Chugh,( May 2015), Review of existing Indian Voting System and hybrid design using Biometric Security in Voting Authentication Process.

4. Shafi'í Muhammad Abdulhamid, Damian Oshomah Ugiomoh, Mohammed Danlami AbdulMalik,(May 2013), The Design and Development of Real-Time E-Voting System in Nigeria with Emphasis on Security and Result Veracity.

5. Sanjay Kumar1, Manpreet Singh2,(July 2013 ), Desigan a secure electronic voting system using fingerpriet technique.

6. B. Madan Mohan Reddy, D. Srihari,( April 2015), RFID Based Biometric Voting Machine Linked To Aadhaar For Safe And Secure Voting.

7. M.Sudhakar, B.Divya Soundarya Sai,( Jan - Feb 2015), Biometric System Based Electronic Voting Machine Using Arm9 Microcontroller. 